\title{
Structural insights into the regulatory mechanism of the Pseudomonas aeruginosa YfiBNR system
}

\author{
Min $\mathrm{Xu}^{1}$, Xuan Yang ${ }^{2}$, Xiu-An Yang ${ }^{1}$, Lei Zhou ${ }^{1,3}$, Tie-Zheng Liu ${ }^{1,3}$, Zusen Fan ${ }^{2 \bowtie}$, Tao Jiang ${ }^{1 \bowtie}$ \\ ${ }^{1}$ National Laboratory of Biomacromolecules, Institute of Biophysics, Chinese Academy of Sciences, Beijing 100101, China \\ 2 Chinese Academy of Sciences Key Laboratory of Infection and Immunity, Institute of Biophysics, Chinese Academy of \\ Sciences, Beijing 100101, China \\ ${ }^{3}$ University of Chinese Academy of Sciences, Beijing 100049, China \\ $\triangle$ Correspondence: fanz@moon.ibp.ac.cn (Z. Fan), tjiang@ibp.ac.cn (T. Jiang) \\ Received February 17, 2016 Accepted March 10, 2016
}

\begin{abstract}
YfiBNR is a recently identified bis-(3'-5')-cyclic dimeric GMP (c-di-GMP) signaling system in opportunistic pathogens. It is a key regulator of biofilm formation, which is correlated with prolonged persistence of infection and antibiotic drug resistance. In response to cell stress, YfiB in the outer membrane can sequester the periplasmic protein YfiR, releasing its inhibition of YfiN on the inner membrane and thus provoking the diguanylate cyclase activity of YfiN to induce c-di-GMP production. However, the detailed regulatory mechanism remains elusive. Here, we report the crystal structures of $Y f i B$ alone and of an active mutant $\mathrm{YfiB}^{\mathrm{L43P}}$ complexed with YfiR with 2:2 stoichiometry. Structural analyses revealed that in contrast to the compact conformation of the dimeric YfiB alone, YfiB ${ }^{\mathrm{L43P}}$ adopts a stretched conformation allowing activated YfiB to penetrate the peptidoglycan (PG) layer and access YfiR. $\mathrm{YfiB}^{\mathrm{L} 43 \mathrm{P}}$ shows a more compact PG-binding pocket and much higher PG binding affinity than wild-type YfiB, suggesting a tight correlation between PG binding and YfiB activation. In addition, our crystallographic analyses revealed that YfiR binds Vitamin B6 (VB6) or L-Trp at a YfiB-binding site and that both VB6 and L-Trp are able to reduce $\mathrm{YfiB}^{\mathrm{L}}{ }^{\mathrm{43P}}$-induced biofilm formation. Based on the structural and biochemical data, we propose an updated regulatory model of the YfiBNR system.
\end{abstract}

Min Xu, Xuan Yang and Xiu-An Yang have contributed equally to this work.
KEYWORDS the YfiBNR system, c-di-GMP, Vitamin B6, L-Trp, peptidoglycan layer, bioflim formation

\section{INTRODUCTION}

Bis-(3'-5')-cyclic dimeric GMP (c-di-GMP) is a ubiquitous second messenger that bacteria use to facilitate behavioral adaptations to their ever-changing environment. An increase in c-di-GMP promotes biofilm formation, and a decrease results in biofilm degradation (Boehm et al., 2010; Duerig et al., 2009; Hickman et al., 2005; Jenal, 2004; Romling et al., 2013). The c-di-GMP level is regulated by two reciprocal enzyme systems, namely, diguanylate cyclases (DGCs) that synthesize c-di-GMP and phosphodiesterases (PDEs) that hydrolyze c-di-GMP (Kulasakara et al., 2006; Ross et al., 1991; Ross et al., 1987). Many of these enzymes are multiple-domain proteins containing a variable $\mathrm{N}$-terminal domain that commonly acts as a signal sensor or transduction module, followed by the relatively conserved GGDEF motif in DGCs or EAL/HD-GYP domains in PDEs (Hengge, 2009; Navarro et al., 2011; Schirmer and Jenal, 2009). Intriguingly, studies in diverse species have revealed that a single bacterium can have dozens of DGCs and PDEs (Hickman et al., 2005; Kirillina et al., 2004; Kulasakara et al., 2006; Tamayo et al., 2005). In Pseudomonas aeruginosa in particular, 42 genes containing putative DGCs and/or PDEs were identified (Kulasakara et al., 2006). The functional role of a number of downstream effectors of c-di-GMP has been characterized as affecting exopolysaccharide (EPS) production, transcription, motility, and surface attachment (Caly et al., 2015; Camilli and Bassler, 2006; Ha and O'Toole, 2015; Pesavento and Hengge, 2009). However, due to the 
intricacy of c-di-GMP signaling networks and the diversity of experimental cues, the detailed mechanisms by which these signaling pathways specifically sense and integrate different inputs remain largely elusive.

Biofilm formation protects pathogenic bacteria from antibiotic treatment, and c-di-GMP-regulated biofilm formation has been extensively studied in $P$. aeruginosa (Evans, 2015; Kirisits et al., 2005; Malone, 2015; Reinhardt et al., 2007). In the lungs of cystic fibrosis (CF) patients, adherent biofilm formation and the appearance of small colony variant (SCV) morphologies of $P$. aeruginosa correlate with prolonged persistence of infection and poor lung function (Govan and Deretic, 1996; Haussler et al., 1999; Haussler et al., 2003; Parsek and Singh, 2003; Smith et al., 2006). Recently, Malone and coworkers identified the tripartite c-diGMP signaling module system YfiBNR (also known as AwsXRO (Beaumont et al., 2009; Giddens et al., 2007) or Tbp (Ueda and Wood, 2009)) by genetic screening for mutants that displayed SCV phenotypes in $P$. aeruginosa PAO1 (Malone et al., 2012; Malone et al., 2010). The YfiBNR system contains three protein members and modulates intracellular c-di-GMP levels in response to signals received in the periplasm (Malone et al., 2010). More recently, this system was also reported in other Gram-negative bacteria, such as Escherichia coli (Hufnagel et al., 2014; Raterman et al., 2013; Sanchez-Torres et al., 2011), Klebsiella pneumonia (Huertas et al., 2014) and Yersinia pestis (Ren et al., 2014). YfiN is an integral inner-membrane protein with two potential transmembrane helices, a periplasmic Per-ArntSim (PAS) domain, and cytosolic domains containing a HAMP domain (mediate input-output signaling in histidine kinases, adenylyl cyclases, methyl-accepting chemotaxis proteins, and phosphatases) and a C-terminal GGDEF domain indicating a DGC's function (Giardina et al., 2013; Malone et al., 2010). YfiN is repressed by specific interaction between its periplasmic PAS domain and the periplasmic protein YfiR (Malone et al., 2010). YfiB is an OmpA/Pal-like outer-membrane lipoprotein (Parsons et al., 2006) that can activate $\mathrm{YfiN}$ by sequestering $\mathrm{YfiR}$ (Malone et al., 2010) in an unknown manner. Whether $\mathrm{YfiB}$ directly recruits $\mathrm{YfiR}$ or recruits $Y f i R$ via a third partner is an open question. After the sequestration of YfiR by $\mathrm{YfiB}$, the c-di-GMP produced by activated YfiN increases the biosynthesis of the Pel and Psl EPSs, resulting in the appearance of the SCV phenotype, which indicates enhanced biofilm formation (Malone et al., 2010).

It has been reported that the activation of YfiN may be induced by redox-driven misfolding of YfiR (Giardina et al., 2013; Malone et al., 2012; Malone et al., 2010). It is also proposed that the sequestration of $\mathrm{YfiR}$ by $\mathrm{YfiB}$ can be induced by certain YfiB-mediated cell wall stress, and mutagenesis studies revealed a number of activation residues of $\mathrm{YfiB}$ that were located in close proximity to the predicted first helix of the periplasmic domain (Malone et al., 2012). In addition, quorum sensing-related dephosphorylation of the PAS domain of YfiN may also be involved in the regulation (Ueda and Wood, 2009; Xu et al., 2015). Recently, we solved the crystal structure of $\mathrm{YfiR}$ in both the non-oxidized and the oxidized states, revealing breakage/formation of one disulfide bond (Cys71-Cys110) and local conformational change around the other one (Cys145-Cys152), indicating that Cys145-Cys152 plays an important role in maintaining the correct folding of YfiR (Yang et al., 2015).

In the present study, we solved the crystal structures of an $\mathrm{N}$-terminal truncated form of YfiB (34-168) and YfiR in complex with an active mutant $\mathrm{YfiB}^{\mathrm{L} 43 \mathrm{P}}$. Most recently, $\mathrm{Li}$ and coworkers reported the crystal structures of YfiB (27-168) alone and $\mathrm{YfiR}^{\mathrm{C71S}}$ in complex with YfiB (59-168) (Li et al., 2015). Compared with the reported complex structure, $\mathrm{YfiB}^{\mathrm{L} 43 \mathrm{P}}$ in our YfiB-YfiR complex structure has additional visible N-terminal residues 44-58 that are shown to play essential roles in YfiB activation and biofilm formation. Therefore, we are able to visualize the detailed allosteric arrangement of the $\mathrm{N}$-terminal structure of $\mathrm{YfiB}$ and its important role in YfiB-YfiR interaction. In addition, we found that the $\mathrm{YfiB}^{\mathrm{L} 43 \mathrm{P}}$ shows a much higher PG-binding affinity than wild-type $Y f i B$, most likely due to its more compact PGbinding pocket. Moreover, we found that Vitamin B6 (VB6) or L-Trp can bind YfiR with an affinity in the ten millimolar range. Together with functional data, these results provide new mechanistic insights into how activated $Y$ fiB sequesters YfiR and releases the suppression of YfiN. These findings may facilitate the development and optimization of anti-biofilm drugs for the treatment of chronic infections.

\section{RESULTS}

\section{Overall structure of $\mathrm{YfiB}$}

We obtained two crystal forms of YfiB (residues 34-168, lacking the signal peptide from residues 1-26 and periplasmic residues 27-33), crystal forms I and II, belonging to space groups $\mathrm{P} 2{ }_{1}$ and $\mathrm{P} 4_{1}$, respectively.

The crystal structure of YfiB monomer consists of a fivestranded $\beta$-sheet ( $\beta 1-2-5-3-4)$ flanked by five $\alpha$-helices $(\alpha 1-5)$ on one side. In addition, there is a short helix turn connecting the $\beta 4$ strand and $\alpha 4$ helix (Fig. 1A and 1B). Each crystal form contains three different dimeric types of $\mathrm{YfiB}$, two of which are present in both, suggesting that the rest of the dimeric types may result from crystal packing. Here, we refer to the two dimeric types as "head to head" and "back to back" according to the interacting mode (Fig. $2 \mathrm{~A}$ and $2 \mathrm{E}$ ), with the total buried surface areas being $316.8 \AA^{2}$ and $554.3 \AA^{2}$, respectively.

The "head to head" dimer exhibits a clamp shape. The dimerization occurs mainly via hydrophobic interactions formed by A37 and 140 on the $\alpha 1$ helices, L50 on the $\beta 1$ strands, and W55 on the $\beta 2$ strands of both molecules, making a hydrophobic interacting core (Fig. 2A-C).

The "back to back" dimer presents a $Y$ shape. The dimeric interaction is mainly hydrophilic, occurring among the main- 
A

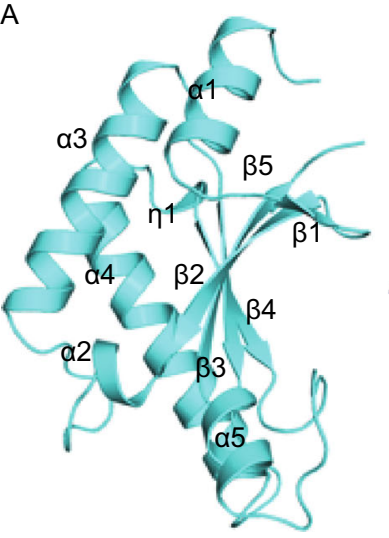

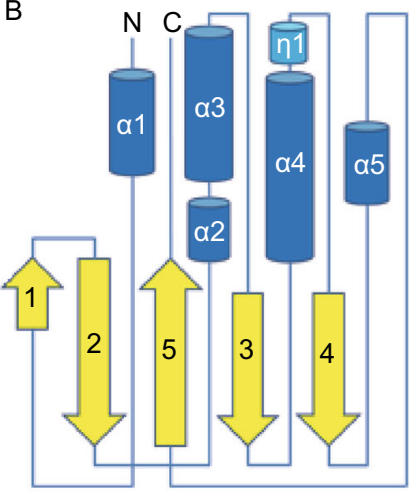

C

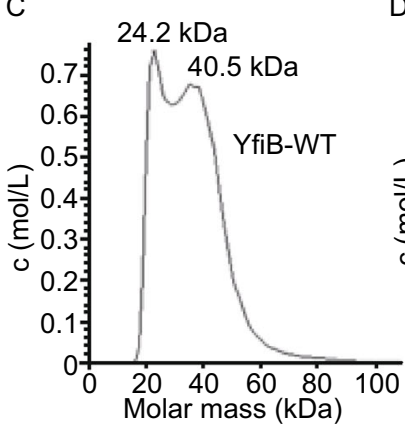

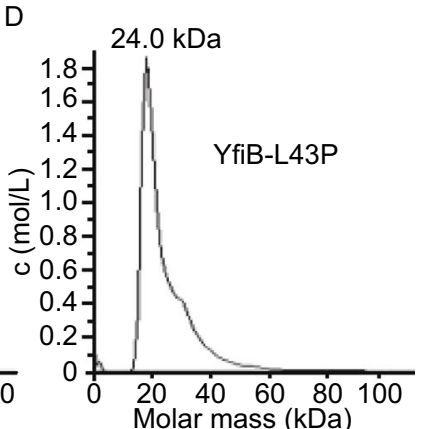

Figure 1. Overall structure of YfiB. (A) The overall structure of the YfiB monomer. (B) A topology diagram of the YfiB monomer. (C and D) The analytical ultracentrifugation experiment results for the wild-type $Y$ fiB and $\mathrm{YfiB}^{\mathrm{L43P}}$.

A

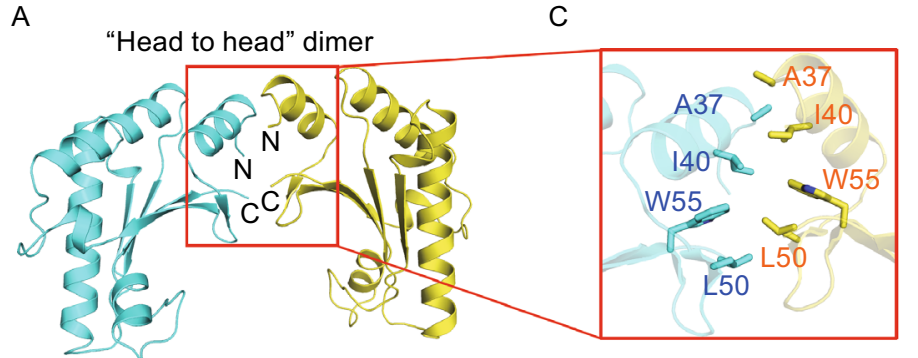

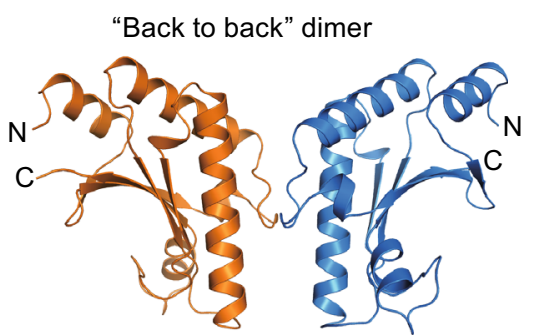

B

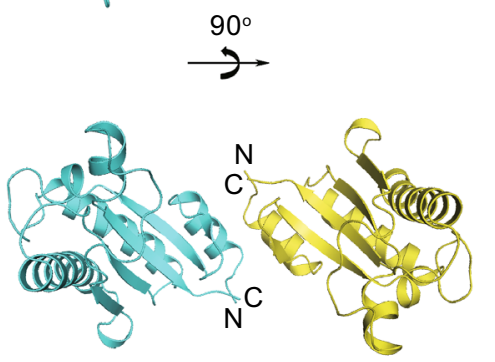

D

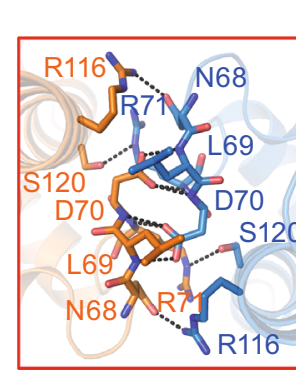

F

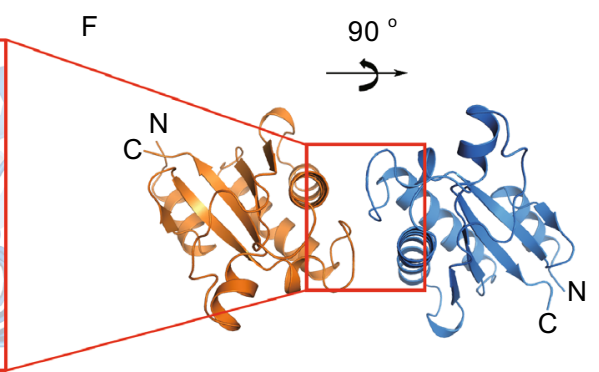

Figure 2. Two dimeric types of YfiB dimer. (A-C) The "head to head" dimer. (D-F) The "back to back" dimer. (A) and (E) indicate the front views of the two dimers, $(B)$ and $(F)$ indicate the top views of the two dimers, and $(C)$ and $(D)$ indicate the details of the two dimeric interfaces.

chain and side-chain atoms of N68, L69, D70 and R71 on the $\alpha 2-\alpha 3$ loops and R116 and S120 on the $\alpha 4$ helices of both molecules, resulting in a complex hydrogen bond network (Fig. 2D-F).

\section{The YfiB-YfiR interaction}

To gain structural insights into the YfiB-YfiR interaction, we co-expressed YfiB (residues 34-168) and YfiR (residues 35-190, lacking the signal peptide), but failed to obtain the complex, in accordance with a previous report in which no stable complex of YfiB-YfiR was observed (Malone et al., 2012). It has been reported that single mutants of Q39, L43, F48 and W55 contribute to YfiB activation leading to the induction of the SCV phenotype in $P$. aeruginosa PAO1 (Malone et al., 2012). It is likely that these residues may be involved in the conformational changes of YfiB that are related to YfiR sequestration (Fig. 3C). Therefore, we constructed two such single mutants of $\mathrm{YfiB}\left(\mathrm{YfiB}^{\mathrm{L43P}}\right.$ and $\mathrm{YfiB}^{\mathrm{F} 48 \mathrm{~S}}$ ). As expected, both mutants form a stable complex with YfiR. Finally, we crystalized YfiR in complex with the $\mathrm{YfiB}^{\mathrm{L} 43 \mathrm{P}}$ mutant and solved the structure at $1.78 \AA$ resolution by molecular replacement using YfiR and YfiB as models.

The YfiB-YfiR complex is a 2:2 heterotetramer (Fig. $3 \mathrm{~A}$ ) in which the YfiR dimer is clamped by two separated $\mathrm{YfiB}^{\mathrm{L43P}}$ molecules with a total buried surface area of $3161.2 \AA^{2}$. The YfiR dimer in the complex is identical to the non-oxidized YfiR dimer alone (Yang et al., 2015), with only Cys145- 


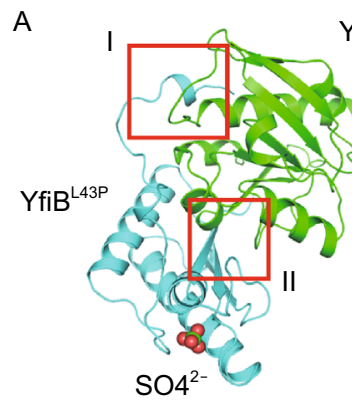

D

I (i)

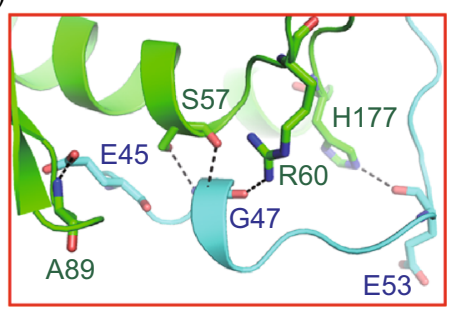

B

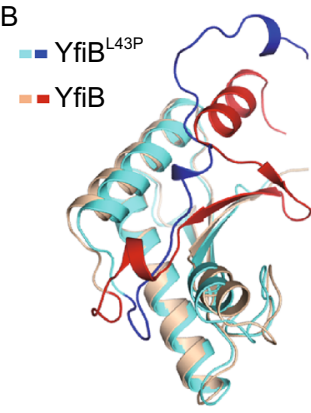

I (ii)

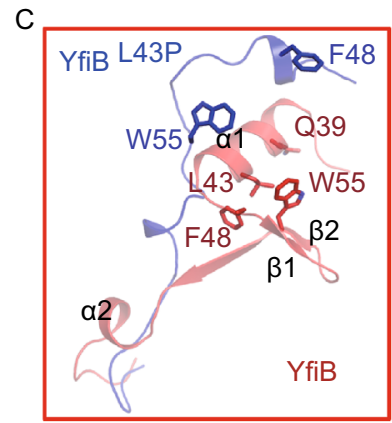

II
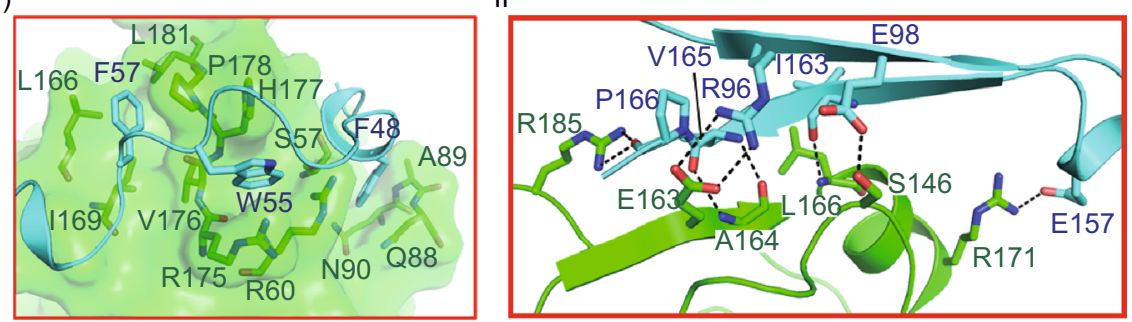

E

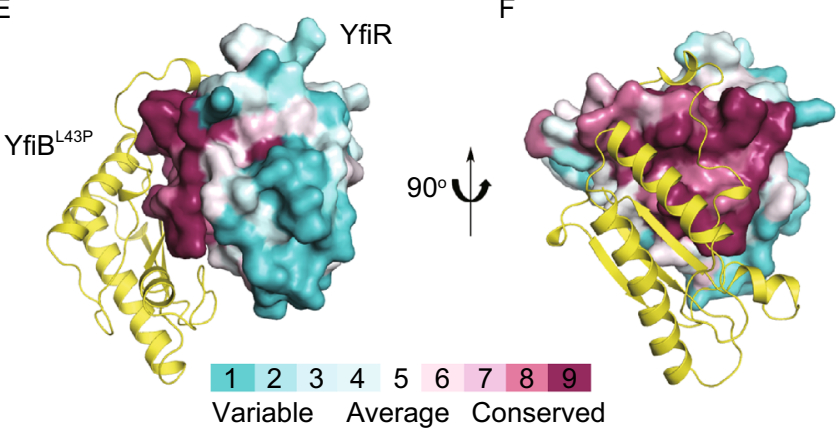

G

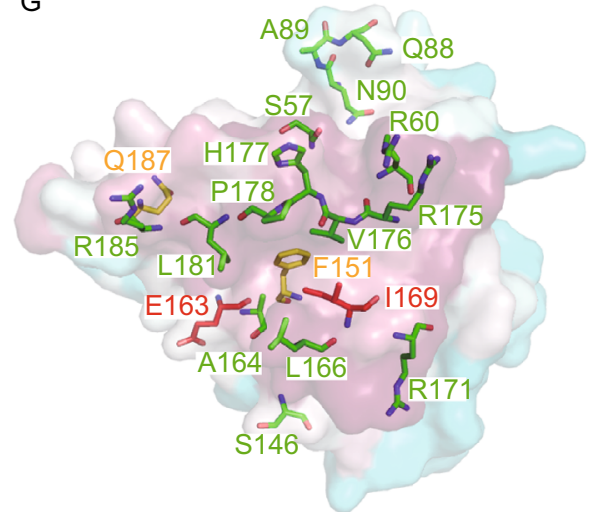

Figure 3. Overall structure of the YfiB-YfiR complex and the conserved surface in YfiR. (A) The overall structure of the YfiBYfiR complex. The YfiB ${ }^{\mathrm{L} 43 \mathrm{P}}$ molecules are shown in cyan and yellow. The YfiR molecules are shown in green and magenta. Two interacting regions are highlighted by red rectangles. (B) Structural superposition of apo YfiB and YfiR-bound YfiB ${ }^{443 P}$. To illustrate the differences between apo YfiB and YfiR-bound $\mathrm{YfiB}^{\mathrm{L43P}}$, the apo YfiB is shown in pink, except residues $34-70$ are shown in red, whereas the YfiR-bound $\mathrm{YfiB}^{\mathrm{L43P}}$ is shown in cyan, except residues 44-70 are shown in blue. (C) Close-up view of the differences between apo YfiB and YfiR-bound YfiB ${ }^{\mathrm{L43P}}$. The residues proposed to contribute to YfiB activation are illustrated in sticks. The key residues in apo YfiB are shown in red and those in $\mathrm{YfiB}^{\mathrm{L43P}}$ are shown in blue. (D) Close-up views showing interactions in regions I and II. YfiB ${ }^{\mathrm{L} 43 \mathrm{P}}$ and YfiR are shown in cyan and green, respectively. (E and $F$ ) The conserved surface in YfiR contributes to the interaction with YfiB. (G) The residues of YfiR responsible for interacting with YfiB are shown in green sticks, and the proposed YfiNinteracting residues are shown in yellow sticks. The red sticks, which represent the YfiB-interacting residues, are also responsible for the proposed interactions with YfiN.

Cys152 of the two disulfide bonds well formed, suggesting Cys71-Cys110 disulfide bond formation is not essential for forming YfiB-YfiR complex. The N-terminal structural conformation of $\mathrm{YfiB}^{\mathrm{L}}{ }^{\mathrm{L} 3 \mathrm{P}}$, from the foremost $\mathrm{N}$-terminus to residue $\mathrm{D70}$, is significantly altered compared with that of the apo YfiB. The majority of the a1 helix (residues 34-43) is invisible on the electron density map, and the $\alpha 2$ helix and $\beta 1$ and $\beta 2$ strands are rearranged to form a long loop containing two short $\alpha$-helix turns (Fig. $3 \mathrm{~B}$ and $3 \mathrm{C}$ ), thus embracing the YfiR dimer. The observed changes in conformation of $\mathrm{YfiB}$ and the results of mutagenesis suggest a mechanism by which $\mathrm{YfiB}$ sequesters YfiR.

The YfiB-YfiR interface can be divided into two regions (Fig. 3A and 3D). Region I is formed by numerous mainchain and side-chain hydrophilic interactions between residues E45, G47 and E53 from the N-terminal extended loop of $\mathrm{YfiB}$ and residues S57, R60, A89 and $\mathrm{H} 177$ from YfiR (Fig. 3D-I(i)). Additionally, three hydrophobic anchoring sites exist in region I. The residues $\mathrm{F} 48$ and W55 of YfiB are inserted into the hydrophobic cores mainly formed by the 
main chain and side chain carbon atoms of residues S57/ Q88/A89/N90 and R60/R175/H177 of YfiR, respectively; and $\mathrm{F} 57$ of $\mathrm{YfiB}$ is inserted into the hydrophobic pocket formed by L166/I169/N176/P178/L181 of YfiR (Fig. 3D-I(ii)). In region II, the side chains of R96, E98 and E157 from YfiB interact with the side chains of E163, S146 and R171 from YfiR, respectively. Additionally, the main chains of I163 and V165 from YfiB form hydrogen bonds with the main chains of L166 and $\mathrm{A} 164$ from $\mathrm{YfiR}$, respectively, and the main chain of $\mathrm{P} 166$ from $\mathrm{YfiB}$ interacts with the side chain of R185 from YfiR (Fig. 3D-II). These two regions contribute a robust hydrogen-bonding network to the YfiB-YfiR interface, resulting in a tightly bound complex.

Based on the observations that two separated $\mathrm{YfiB}^{\mathrm{L43P}}$ molecules form a 2:2 complex structure with YfiR dimer, we performed an analytical ultracentrifugation experiment to check the oligomeric states of wild-type $Y$ fiB and $\mathrm{YfiB}^{\mathrm{L}}{ }^{\mathrm{L} P}$. The results showed that wild-type $Y f i B$ exists in both monomeric and dimeric states in solution, while $\mathrm{YfiB}^{\mathrm{L} 43 \mathrm{P}}$ primarily adopts the monomer state in solution (Fig. 1C-D). This suggests that the $\mathrm{N}$-terminus of $\mathrm{YfiB}$ plays an important role in forming the dimeric $\mathrm{YfiB}$ in solution and that the conformational change of residue L43 is associated with the stretch of the $\mathrm{N}$-terminus and opening of the dimer. Therefore, it is possible that both dimeric types might exist in solution. For simplicity, we only discuss the "head to head" dimer in the following text.

\section{The PG-binding site of YfiB}

PG-associated lipoprotein (Pal) is highly conserved in Gramnegative bacteria and anchors to the outer membrane through an N-terminal lipid attachment and to $P G$ layer through its periplasmic domain, which is implicated in maintaining outer membrane integrity. Previous homology modeling studies suggested that YfiB contains a Pal-like PGbinding site (Parsons et al., 2006), and the mutation of two residues at this site, D102 and G105, reduces the ability for biofilm formation and surface attachment (Malone et al., 2012). In the YfiB-YfiR complex, one sulfate ion is found at the bottom of each $\mathrm{YfiB}^{\mathrm{L} 43 \mathrm{P}}$ molecule (Fig. $3 \mathrm{~A}$ ) and forms a strong hydrogen bond with $\mathrm{D} 102$ of $\mathrm{YfiB}^{\mathrm{L43P}}$ (Fig. 4A and $4 C)$. Structural superposition between $\mathrm{YfiB}^{\mathrm{L} 43 \mathrm{P}}$ and Haemophilus influenzae Pal complexed with biosynthetic peptidoglycan precursor (PG-P), UDP-N-acetylmuramyl-L-Ala- $\alpha-$ D-Glu-m-Dap-D-Ala-D-Ala ( $m$-Dap is meso-diaminopimelate) (PDB code: 2aiz) (Parsons et al., 2006), revealed that the sulfate ion is located at the position of the m-Dap5 $\epsilon$-carboxylate group in the Pal/PG-P complex (Fig. 4A). In the Pal/PG-P complex structure, the m-Dap5 $\epsilon$-carboxylate group interacts with the side-chain atoms of D71 and the main-chain amide of D37 (Fig. 4B). Similarly, in the YfiRbound $\mathrm{YfiB}^{\mathrm{L43P}}$ structure, the sulfate ion interacts with the side-chain atoms of D102 (corresponding to D71 in Pal) and R117 (corresponding to R86 in Pal) and the main-chain amide of $\mathrm{N} 68$ (corresponding to D37 in Pal). Moreover, a water molecule was found to bridge the sulfate ion and the side chains of N67 and D102, strengthening the hydrogen bond network (Fig. 4C). In addition, sequence alignment of $\mathrm{YfiB}$ with $\mathrm{Pal}$ and the periplasmic domain of OmpA (proteins containing PG-binding site) showed that N68 and D102 are highly conserved (Fig. 4G, blue stars), suggesting that these residues contribute to the $P G$-binding ability of YfiB.

Interestingly, superposition of apo YfiB with YfiR-bound $\mathrm{YfiB}^{\mathrm{L} 43 \mathrm{P}}$ revealed that the $\mathrm{PG}$-binding region is largely altered mainly due to different conformation of the N68 containing loop. Compared to $\mathrm{YfiB}^{\mathrm{L43P}}$, the N68-containing loop of the apo YfiB flips away about $7 \AA$, and D102 and R117 swing slightly outward; thus, the PG-binding pocket is enlarged with no sulfate ion or water bound (Fig. 4D). Therefore, we proposed that the PG-binding ability of inactive YfiB might be weaker than that of active YfiB. To validate this, we performed a microscale thermophoresis (MST) assay to measure the binding affinities of $P G$ to wild-type $\mathrm{YfiB}$ and $\mathrm{YfiB}^{\mathrm{L}}{ }^{\mathrm{L} P \mathrm{P}}$, respectively. The results indicated that the $P G-b i n d i n g$ affinity of $Y f i B^{L 43 P}$ is $65.5 \mu \mathrm{mol} / \mathrm{L}$, which is about 16 -fold stronger than that of wild-type $\mathrm{YfiB}\left(K_{\mathrm{d}}=1.1 \mathrm{mmol} / \mathrm{L}\right)$ (Fig. 4E-F). As the experiment is performed in the absence of $Y f i R$, it suggests that an increase in the PG-binding affinity of $Y$ fiB is not a result of $Y f i B-Y f i R$ interaction and is highly coupled to the activation of $\mathrm{YfiB}$ characterized by a stretched $\mathrm{N}$-terminal conformation.

The conserved surface in YfiR is functional for binding YfiB and YfiN

Calculation using the ConSurf Server (http://consurf.tau.ac.il/), which estimates the evolutionary conservation of amino acid positions and visualizes information on the structure surface, revealed a conserved surface on $\mathrm{YfiR}$ that contributes to the interaction with YfiB (Fig. 3E and 3F). Interestingly, the majority of this conserved surface contributes to the interaction with $\mathrm{YfiB}$ (Fig. 3E and 3F). Malone JG et al. have reported that F151, E163, I169 and Q187, located near the C-terminus of $\mathrm{YfiR}$, comprise a putative YfiN binding site (Malone et al., 2012). Interestingly, these residues are part of the conserved surface of YfiR (Fig. 3G). F151, E163 and I169 form a hydrophobic core while, Q187 is located at the end of the $a 6$ helix. E163 and I169 are YfiB-interacting residues of YfiR, in which E163 forms a hydrogen bond with R96 of $\mathrm{YfiB}$ (Fig. 3D-II) and I169 is involved in forming the L166/I169/ V176/P178/L181 hydrophobic core for anchoring F57 of YfiB (Fig. 3D-I(ii)). Collectively, a part of the YfiB-YfiR interface overlaps with the proposed YfiR-YfiN interface, suggesting alteration in the association-disassociation equilibrium of YfiR-YfiN and hence the ability of YfiB to sequester YfiR.

\section{YfiR binds small molecules}

Previous studies indicated that $Y$ fiR constitutes a YfiB-independent sensing device that can activate $\mathrm{YfiN}$ in response to 

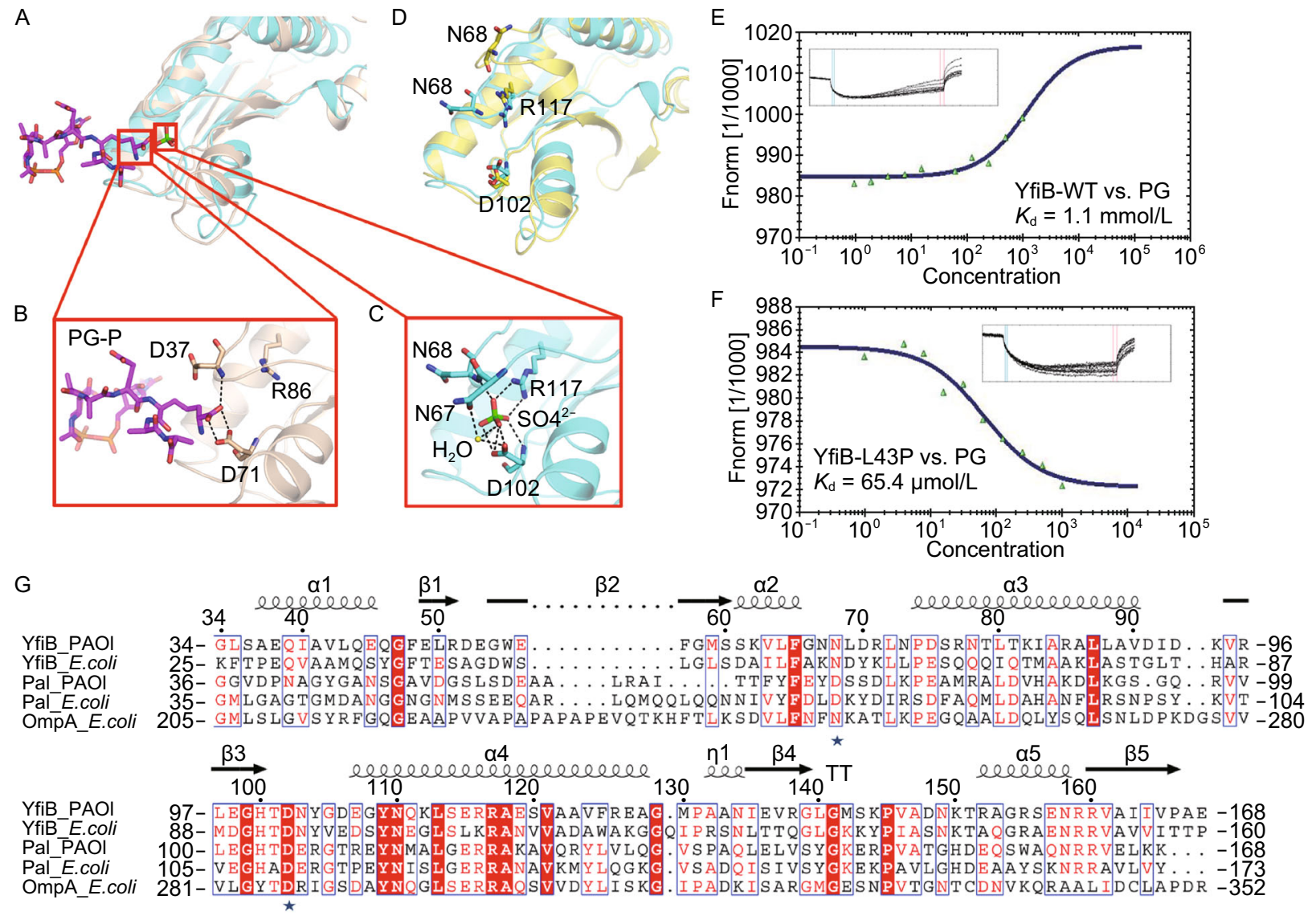

Figure 4. The PG-binding site in YfiB. (A) Structural superposition of the PG-binding sites of the $H$. influenzae Pal/PG-P complex and $Y$ fiR-bound $Y f i B^{L 43 P}$ complexed with sulfate ions. (B) Close-up view showing the key residues of Pal interacting with the $m$-Dap5 $\varepsilon$-carboxylate group of PG-P. Pal is shown in wheat and PG-P is in magenta. (C) Close-up view showing the key residues of YfiRbound $\mathrm{YfiB}^{\mathrm{L} 43 \mathrm{P}}$ interacting with a sulfate ion. $\mathrm{YfiR}$-bound $\mathrm{YfiB}^{\mathrm{L} 43 \mathrm{P}}$ is shown in cyan; the sulfate ion, in green; and the water molecule, in yellow. (D) Structural superposition of the PG-binding sites of apo YfiB and YfiR-bound $\mathrm{YfiB}^{\mathrm{L43P}}$, the key residues are shown in stick. Apo $Y f i B$ is shown in yellow and $Y f i R-$ bound $Y f_{i B}{ }^{L 43 P}$ in cyan. (E and F) MST data and analysis for binding affinities of (E) YfiB wild-type and $(\mathrm{F}) \mathrm{YfiB}^{\mathrm{L} 43 \mathrm{P}}$ with $\mathrm{PG}$. (G) The sequence alignment of $P$. aeruginosa and $E$. coli sources of $Y \mathrm{fiB}$, Pal and the periplasmic domain of OmpA.

the redox status of the periplasm, and we have reported YfiR structures in both the non-oxidized and the oxidized states earlier, revealing that the Cys145-Cys 152 disulfide bond plays an essential role in maintaining the correct folding of YfiR (Yang et al., 2015). However, whether YfiR is involved in other regulatory mechanisms is still an open question.

Intriguingly, a Dali search (Holm and Rosenstrom, 2010) indicated that the closest homologs of YfiR shared the characteristic of being able to bind several structurally similar small molecules, such as L-Trp, L-Phe, B-group vitamins and their analogs, encouraging us to test whether YfiR can recognize these molecules. For this purpose, we co-crystallized YfiR or soaked YfiR crystals with different small molecules, including L-Trp and B-group vitamins. Fortunately, we found obvious small-molecule density in the VB6-bound and Trpbound YfiR crystal structures (Fig. 5A and 5B), and in both structures, the $\mathrm{YfiR}$ dimers resemble the oxidized $\mathrm{YfiR}$ structure in which both two disulfide bonds are well formed (Yang et al., 2015).

Structural analyses revealed that the VB6 and L-Trp molecules are bound at the periphery of the YfiR dimer, but not at the dimer interface. Interestingly, VB6 and L-Trp were found to occupy the same hydrophobic pocket, formed by L166//169/ V176/P178/L181 of YfiR, which is also a binding pocket for F57 of YfiB, as observed in the YfiB-YfiR complex (Fig. 5C). To evaluate the importance of $\mathrm{F} 57$ in $\mathrm{YfiB}^{\mathrm{L}}{ }^{\mathrm{L} 3 \mathrm{P}}{ }_{-} \mathrm{YfiR}$ interaction, the binding affinities of $Y \mathrm{fiB}^{\mathrm{L43P}}$ and $Y \mathrm{FiB}^{\mathrm{L4}}{ }^{\mathrm{L} P / \mathrm{F} 57 \mathrm{~A}}$ for $Y$ fiR were measured by isothermal titration calorimetry (ITC). The results showed $\mathrm{K}_{\mathrm{d}}$ values of $1.4 \times 10^{-7} \mathrm{~mol} / \mathrm{L}$ and $5.3 \times 10^{-7} \mathrm{~mol} / \mathrm{L}$ for $\mathrm{YfiB}^{\mathrm{L43P}}$ and $\mathrm{YfiB}^{\mathrm{L43P/F57A}}$, respectively, revealing that the $\mathrm{YfiB}^{\mathrm{L43P/F57A}}$ mutant caused a 3.8-fold reduction in the binding affinity compared with the $\mathrm{YfiB}^{\mathrm{L} 43 \mathrm{P}}$ mutant (Fig. $6 \mathrm{~F}$ and $6 \mathrm{G}$ ).

In parallel, to better understand the putative functional role of VB6 and L-Trp, yfiB was deleted in a PAO1 wild-type 
A

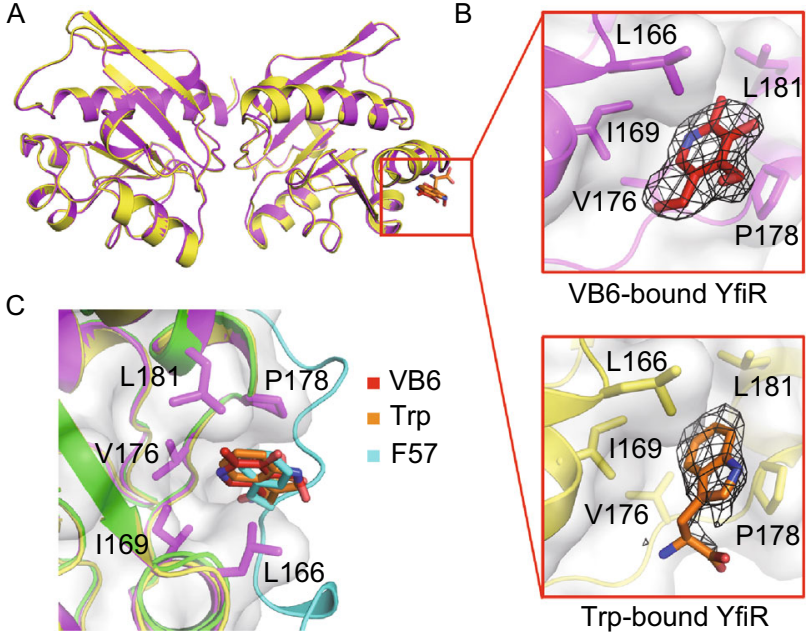

Figure 5. Overall Structures of VB6-bound and Trp-bound YfiR. (A) Superposition of the overall structures of VB6-bound and Trp-bound YfiR. (B) Close-up views showing the key residues of YfiR that bind VB6 and L-Trp. The electron densities of VB6 and Trp are countered at 3.0 0 and $2.3 \sigma$, respectively, in $\left|F_{\mathrm{O}}\right|-\left|F_{\mathrm{C}}\right|$ maps. (C) Superposition of the hydrophobic pocket of YfiR with VB6, L-Trp and F57 of YfiB.

strain, and a construct expressing the $\mathrm{YfiB}^{\mathrm{L43P}}$ mutant was transformed into the PAO1 $\triangle y f i B$ strain to trigger $\mathrm{YfiB}^{\mathrm{L} 43 \mathrm{P}}$. induced biofilm formation. Growth and surface attachment assays were carried out for the $y f i B$-L43P strain in the presence of increasing concentrations of VB6 or L-Trp. As shown in Fig. $6 \mathrm{~A}$ and $6 \mathrm{~B}$, the over-expression of $\mathrm{YfiB}^{\mathrm{L} 43 \mathrm{P}}$ induced strong surface attachment and much slower growth of the $y f i B$-L43P strain, and as expected, a certain amount of VB6 or L-Trp (4-6 mmol/L for VB6 and $6-10 \mathrm{mmol} / \mathrm{L}$ for L-Trp) could reduce the surface attachment. Interestingly, at a concentration higher than $8 \mathrm{mmol} / \mathrm{L}$, VB6 lost its ability to inhibit biofilm formation, implying that the VB6-involving regulatory mechanism is highly complicated and remains to be further investigated.

Of note, both VB6 and L-Trp have been reported to correlate with biofilm formation in certain Gram-negative bacteria (Grubman et al., 2010; Shimazaki et al., 2012). In Helicobacter pylori in particular, VB6 biosynthetic enzymes act as novel virulence factors, and VB6 is required for full motility and virulence (Grubman et al., 2010). In E. coli, mutants with decreased tryptophan synthesis show greater biofilm formation, and matured biofilm is degraded by L-tryptophan addition (Shimazaki et al., 2012). However, the detailed mechanism remains elusive.

To answer the question whether competition of VB6 or L-Trp for the YfiB F57-binding pocket of YfiR plays an essential role in inhibiting biofilm formation, we measured the binding affinities of VB6 and L-Trp for YfiR via BIAcore experiments. The results showed relatively weak $K_{\mathrm{d}}$ values of $35.2 \mathrm{mmol} / \mathrm{L}$ and $76.9 \mathrm{mmol} / \mathrm{L}$ for VB6 and L-Trp, respectively (Fig. $6 \mathrm{C}$ and $6 \mathrm{D}$ ). Based on our results, we

concluded that VB6 or L-Trp can bind to YfiR, however, VB6 or L-Trp alone may have little effects in interrupting the YfiBYfiR interaction, the mechanism by which VB6 or L-Trp inhibits biofilm formation remains unclear and requires further investigation.

\section{DISCUSSION}

Previous studies suggested that in response to cell stress, $Y \mathrm{YiB}$ in the outer membrane sequesters the periplasmic protein YfiR, releasing its inhibition of YfiN on the inner membrane and thus inducing the diguanylate cyclase activity of YfiN to allow c-di-GMP production (Giardina et al., 2013; Malone et al., 2012; Malone et al., 2010). However, the pattern of interaction between these proteins and the detailed regulatory mechanism remain unknown due to a lack of structural information.

Here, we report the crystal structures of $Y f i B$ alone and an active mutant $\mathrm{YfiB}^{\mathrm{L43P}}$ in complex with $\mathrm{YfiR}$, indicating that YfiR forms a 2:2 complex with YfiB via a region composed of conserved residues. Our structural data analysis shows that the activated $\mathrm{YfiB}$ has an $\mathrm{N}$-terminal portion that is largely altered, adopting a stretched conformation compared with the compact conformation of the apo YfiB. The apo YfiB structure constructed beginning at residue 34 has a compact conformation of approximately $45 \AA$ in length. In addition to the preceding 8 aa loop (from the lipid acceptor Cys26 to Gly34), the full length of the periplasmic portion of apo YfiB can reach approximately $60 \AA$. It was reported that the distance between the outer membrane and the cell wall is approximately $50 \AA$ and that the thickness of the PG layer is approximately $70 \AA$ (Matias et al., 2003). Thus, YfiB alone represents an inactive form that may only partially insert into the PG matrix. By contrast, YfiR-bound YfiB ${ }^{L 43 P}$ (residues 44-168) has a stretched conformation of approximately $55 \AA$ in length. In addition to the 17 preceding intracellular residues (from the lipid acceptor Cys26 to Leu43), the length of the intracellular portion of active YfiB may extend over $100 \AA$, assuming a fully stretched conformation. Provided that the diameter of the widest part of the YfiB dimer is approximately $64 \AA$, which is slightly smaller than the smallest diameter of the PG pore (70 A) (Meroueh et al., 2006), the YfiB dimer should be able to penetrate the PG layer.

These results, together with our observation that activated YfiB has a much higher cell wall binding affinity, and previous mutagenesis data showing that (1) both PG binding and membrane anchoring are required for YfiB activity and (2) activating mutations possessing an altered $\mathrm{N}$-terminal loop length are dominant over the loss of PG binding (Malone et al., 2012), suggest an updated regulatory model of the YfiBNR system (Fig. 7). In this model, in response to a particular cell stress that is yet to be identified, the dimeric $Y$ fiB is activated from a compact, inactive conformation to a stretched conformation, which possesses increased PG binding affinity. This allows the C-terminal portion of the membrane-anchored $\mathrm{YfiB}$ to reach, bind and penetrate the 


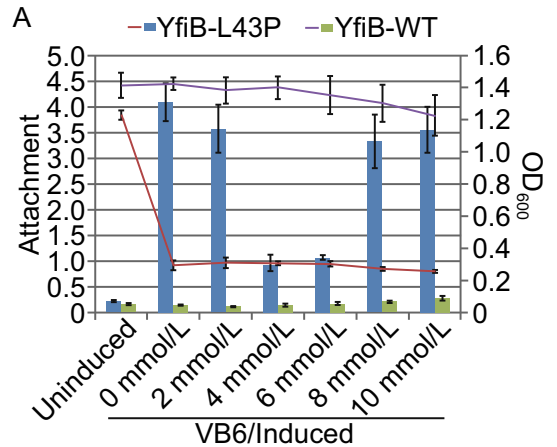

B

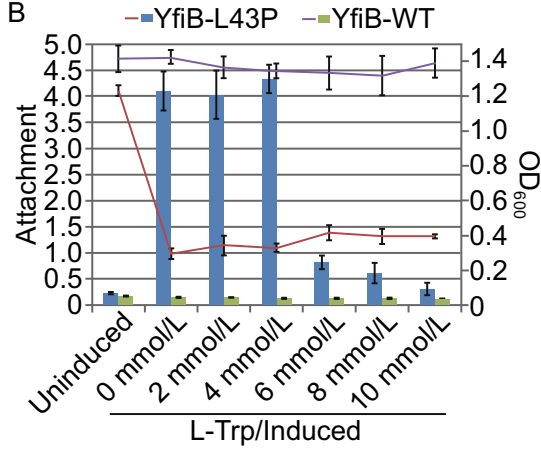

E

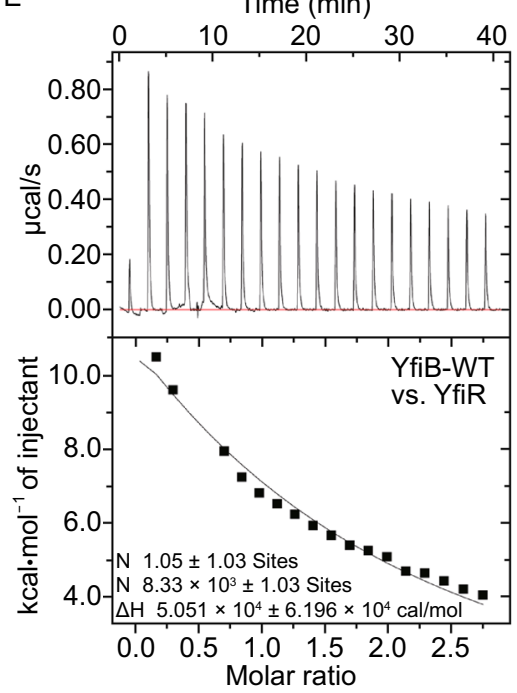

C
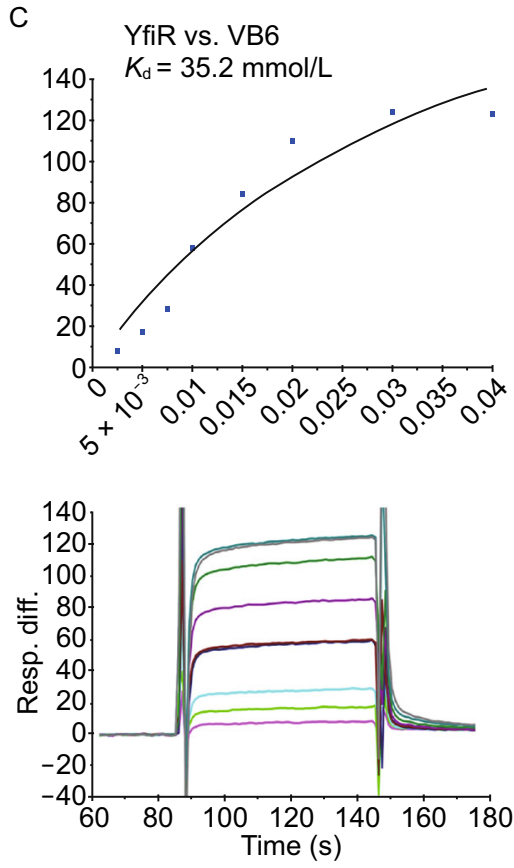

F

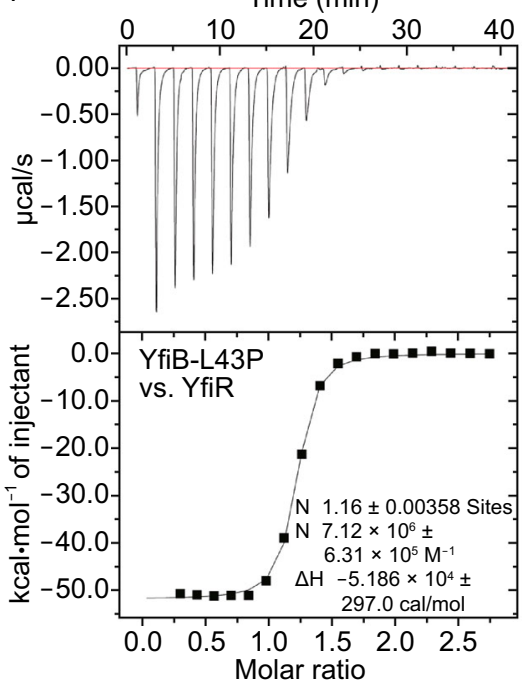

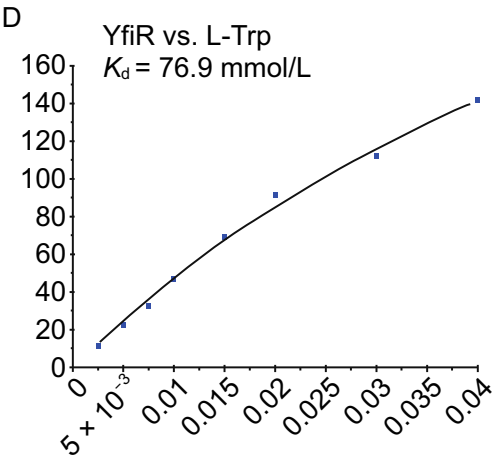

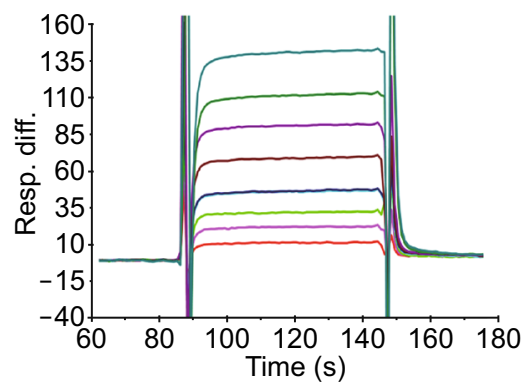

G Time (min)

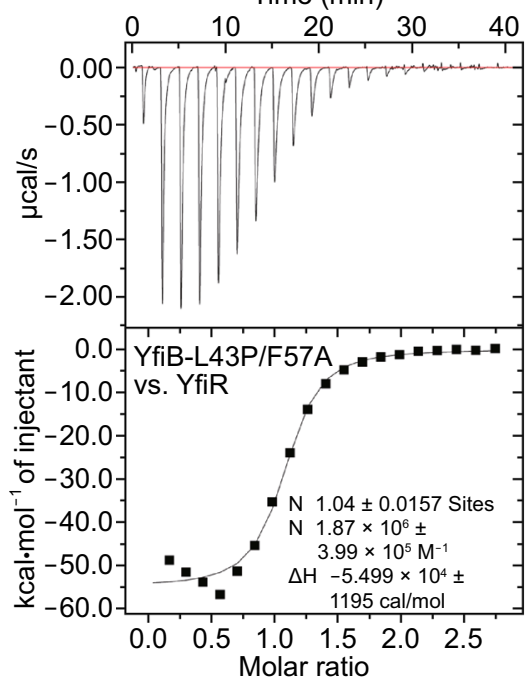

Figure 6. Functional analysis of VB6 and L-Trp. (A and B) The effect of increasing concentrations of VB6 or L-Trp on YfiB ${ }^{\mathrm{L} 43 \mathrm{P}}$ induced attachment (bars). The relative optical density is represented as curves. Wild-type $Y f i B$ is used as negative control. (C and $D)$ BIAcore data and analysis for binding affinities of (C) VB6 and (D) L-Trp with YfiR. (E-G) ITC data and analysis for titration of (E) YfiB wild-type, (F) YfiB ${ }^{\mathrm{L43P}}$, and (G) YfiB ${ }^{\mathrm{L43P/F57A}}$ into YfiR.

cell wall and sequester the YfiR dimer. The YfiBNR system provides a good example of a delicate homeostatic system that integrates multiple signals to regulate the c-di-GMP level. Homologs of the YfiBNR system are functionally conserved in $P$. aeruginosa (Malone et al., 2012; Malone et al., 2010), E. coli (Hufnagel et al., 2014; Raterman et al., 2013; Sanchez-Torres et al., 2011), K. pneumonia (Huertas et al.,
2014) and Y. pestis (Ren et al., 2014), where they affect c-diGMP production and biofilm formation. The mechanism by which activated $\mathrm{YfiB}$ relieves the repression of $\mathrm{YfiN}$ may be applicable to the YfiBNR system in other bacteria and to analogous outside-in signaling for c-di-GMP production, which in turn may be relevant to the development of drugs that can circumvent complicated antibiotic resistance. 
$\mathrm{OM}$
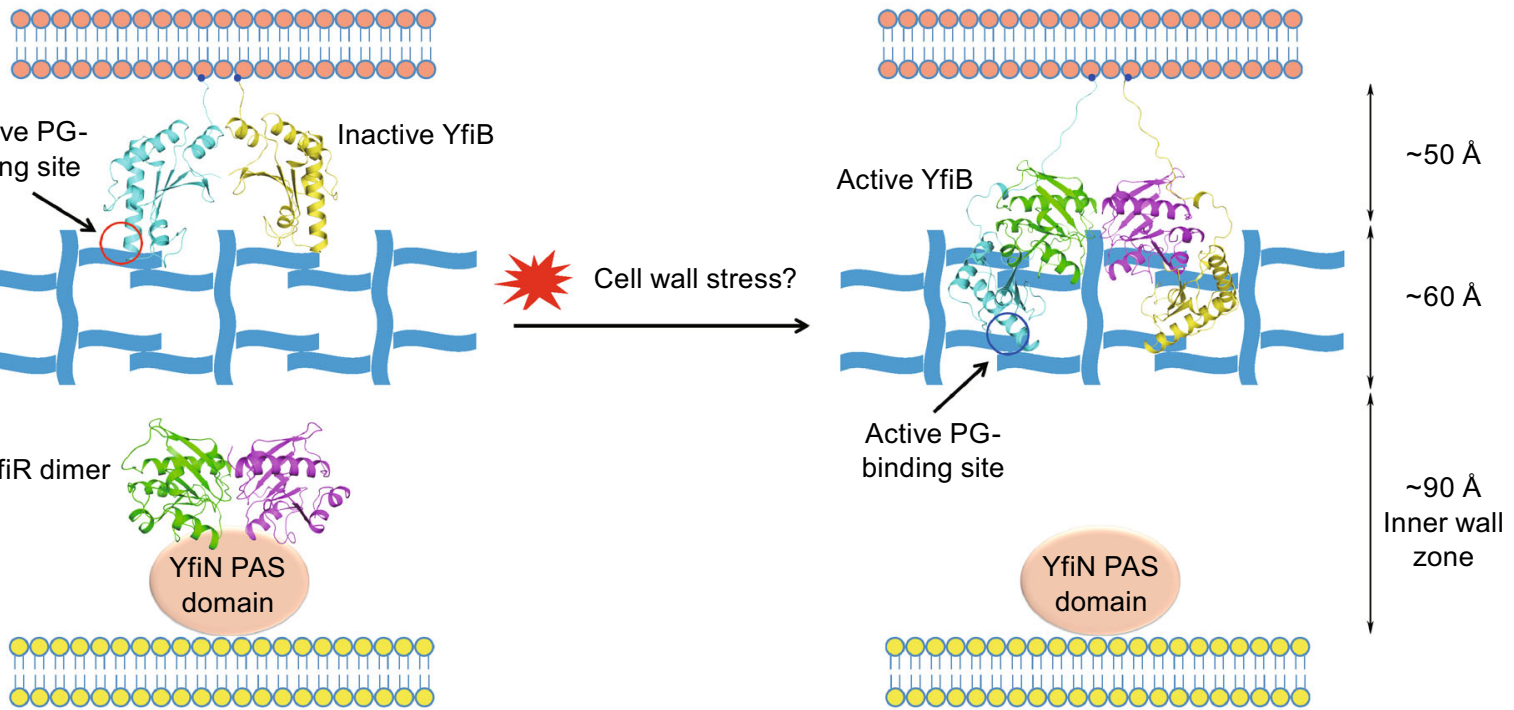

IM

Inactive PG- Inactive YfiB binding site

$P G$
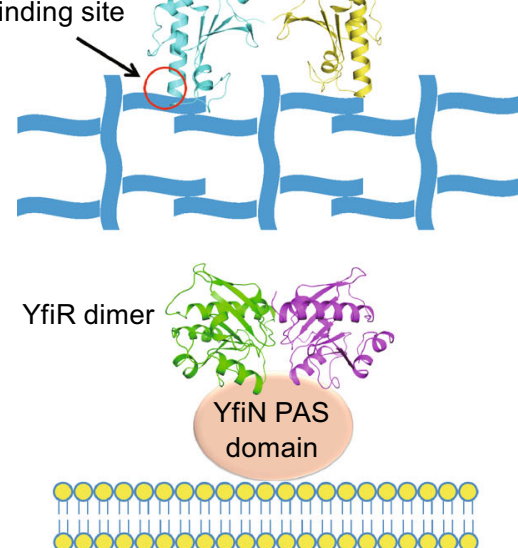

Figure 7. Regulatory model of the YfiBNR tripartite system. The periplasmic domain of YfiB and the YfiB-YfiR complex are depicted according to the crystal structures. The lipid acceptor Cys26 is indicated as blue ball. The loop connecting Cys26 and Gly34 of $\mathrm{YfiB}$ is modeled. The PAS domain of YfiN is shown as pink oval. Once activated by certain cell stress, the dimeric YfiB transforms from a compact conformation to a stretched conformation, allowing the periplasmic domain of the membrane-anchored $Y$ fiB to penetrate the cell wall and sequester the YfiR dimer, thus relieving the repression of YfiN.

\section{MATERIALS AND METHODS}

Protein expression and purification

$P$. aeruginosa YfiR (residues 35-190, lacking the predicted N-terminal periplasmic localization signaling peptide) and YfiB (residues 34-168, lacking the signal peptide from residues 1-26 and periplasmic residues 27-33) were cloned into ORF1 of the pETDuet-1 (Merck Millipore, Darmstadt, Germany) vector via the BamHI and HindIII restriction sites, with a constructed $\mathrm{N}$-terminal $\mathrm{His}_{6}$ and a TEV cleavage site, respectively. In addition, YfiB (residues 34-168) was ligated into the Ndel and Xhol restriction sites of ORF2 in the previously constructed $\mathrm{YfiR}$ expression vector. Site-directed mutagenesis was carried out using a QuikChange kit (Agilent Technologies, Santa Clara, CA), following the manufacturer's instructions.

The proteins were over-expressed in the $E$. coli BL21-CodonPlus (DE3)-RIPL strain. Protein expression was induced by adding $0.5-1 \mathrm{mmol} / \mathrm{L}$ IPTG at an OD600 of approximately 0.8 . The cell cultures were then incubated for an additional $4.5 \mathrm{~h}$ at $37^{\circ} \mathrm{C}$. The cells were subsequently harvested by centrifugation and stored at $-80^{\circ} \mathrm{C}$.

Cell suspensions were thawed and homogenized using a highpressure homogenizer (JNBIO, Beijing, China). YfiR was first purified by $\mathrm{Ni}$ affinity chromatography and then incubated with $\mathrm{His}_{6}{ }^{-}$ tagged TEV protease overnight. The $\mathrm{His}_{6}-\mathrm{TEV}$ cleavage site was subsequently removed by incubation with Ni-NTA resin. Finally, YfiR was purified with a HiTrap $S^{\mathrm{TM}}$ column (GE Healthcare), followed by a Superdex 200 (GE Healthcare) column. YfiB was purified with $\mathrm{Ni}$ affinity chromatography, followed by a Superdex 200 (GE Healthcare) column. The YfiB-YfiR complex was first purified by Ni affinity chromatography, then by a Superdex 200 (GE Healthcare) column, and finally by a HiTrap $S^{T M}$ column (GE Healthcare). All of the purified fractions were collected and concentrated to $\sim 40 \mathrm{mg} / \mathrm{mL}$ in
$20 \mathrm{mmol} / \mathrm{L}$ Tris- $\mathrm{HCl}(\mathrm{pH} 8.0)$ and $200 \mathrm{mmol} / \mathrm{L} \mathrm{NaCl}$, frozen in liquid nitrogen and stored at $-80^{\circ} \mathrm{C}$.

\section{Crystallization and data collection}

Crystal screening was performed with commercial screening kits (Hampton Research, CA, USA) using the sitting-drop vapor diffusion method, and positive hits were optimized using the hanging-drop vapor diffusion method at $293 \mathrm{~K}$. Crystals of the YfiB protein were obtained and optimized in buffer containing $0.2 \mathrm{~mol} / \mathrm{L}$ lithium sulfate monohydrate, $0.1 \mathrm{~mol} / \mathrm{L}$ Tris- $\mathrm{HCl}(\mathrm{pH} 8.0)$ and $30 \% \mathrm{w} / \mathrm{v}$ polyethylene glycol 4000 . After being soaked for a few seconds in cryoprotection solution (well solution complemented with $25 \%$ xylitol), the crystals were cooled by plunging them into liquid nitrogen. Diffraction-quality crystals of the YfiB-YfiR complex were grown in buffer containing $0.2 \mathrm{~mol} / \mathrm{L}$ ammonium sulfate, $0.1 \mathrm{~mol} / \mathrm{L}$ Tris- $\mathrm{HCl}(\mathrm{pH} 8.0)$ and $12 \%$ $w / v$ polyethylene glycol 8000 . The crystals were cryoprotected with $8 \%(w / v)$ polyethylene glycol 8000 and $0.1 \mathrm{~mol} / \mathrm{L}$ Tris- $\mathrm{HCl}(\mathrm{pH} 7.5)$ supplemented with saturated sucrose prior to being flash frozen. Crystals of the native $\mathrm{YfiR}$ were obtained and optimized in $0.1 \mathrm{~mol} / \mathrm{L}$ HEPES ( $\mathrm{pH} 7.5$ ) and $1.8 \mathrm{~mol} / \mathrm{L}$ ammonium sulfate. VB6-bound YfiR crystals were obtained by soaking the native $Y$ fiR crystals in $2 \mathrm{mmol} / \mathrm{L}$ VB6 molecules. Trp-bound YfiR crystals were obtained by co-crystalizing the $\mathrm{YfiR}$ protein and $4 \mathrm{mmol} / \mathrm{L}$ L-Trp molecules in $0.2 \mathrm{~mol} / \mathrm{L} \mathrm{NaCl}, 0.1 \mathrm{~mol} / \mathrm{L}$ BIS-TRIS ( $\mathrm{pH} \mathrm{5.5)}$, and $25 \% \mathrm{w} / \mathrm{v}$ polyethylene glycol 3350 . For cryoprotection, both the VB6-bound and the L-Trp-bound YfiR crystals were soaked in $2.5 \mathrm{~mol} / \mathrm{L}$ lithium sulfate monohydrate for a few seconds before data collection. Diffraction data for the $\mathrm{YfiB}$ crystal belonging to space group $\mathrm{P} 2_{1}$ was collected in house, the data for the YfiB crystal belonging to space group $\mathrm{P} 4_{1}$ and for the Trp-bound YfiR crystal were collected on 
beamline BL17U at the Shanghai Synchrotron Radiation Facility (SSRF), and the data for the VB6-bound YfiR crystal were collected on beamline BL18U at SSRF. Finally, the data for the YfiB-YfiR complex crystal were collected on beamline BL-1A at the Photon Factory in Japan. The diffraction data were processed with the HKL2000 software program (Otwinowski and Minor, 1997).

\section{Structure determination and refinement}

The two YfiB crystal structures respectively belonging to space groups $\mathrm{P} 2{ }_{1}$ and $\mathrm{P} 4_{1}$ were both solved by molecular replacement (Lebedev et al., 2008) using the putative MotB-like protein DVU_2228 from D. vulgaris as a model (PDB code: $3 \mathrm{khn}$ ) at $2.15 \AA$ and $2.8 \AA$ resolution, respectively. Both the VB6-bound and the Trpbound YfiR crystals belonging to space group $\mathrm{P} 4{ }_{3} 2{ }_{1} 2$, with a dimer in the asymmetric unit, were solved by molecular replacement (Lebedev et al., 2008) using native YfiR as a model (PDB code: 4YN7) at $2.4 \AA$ and $2.5 \AA$ resolution, respectively. The YfiB-YfiR crystal belonging to space group $\mathrm{P} 1$, with a 2:2 heterotetramer in the asymmetric unit, was solved by molecular replacement using YfiR and $\mathrm{YfiB}$ as models. Electron density maps were calculated using PHENIX (Adams et al., 2010). Model building was performed using COOT (Emsley et al., 2010) and refined with PHENIX (Adams et al., 2010; Afonine et al., 2012). The final structures were analyzed with PROCHECK (Laskowski et al., 1993). Data collection and refinement statistics are presented in Table 1. The figures depicting structures were prepared using PyMOL (http://www.pymol.org). Atomic coordinates and structure factors have been deposited in the RCSB Protein Data Bank (http://www.pdb.org) under accession codes 5EAZ, 5EB0, 5EB1, 5EB2 and 5EB3.

\section{Analytical ultracentrifugation}

Sedimentation velocity measurements were performed on a Beckman ProteomeLab XL-I at $25^{\circ} \mathrm{C}$. All protein samples were diluted to an $\mathrm{OD}_{280}$ of 0.7 in $20 \mathrm{mmol} / \mathrm{L}$ Tris $(\mathrm{pH} 8.0)$ and $200 \mathrm{mmol} / \mathrm{L} \mathrm{NaCl}$. Data were collected at $60,000 \mathrm{rpm}$. $(262,000 \times \mathrm{g})$ every $3 \mathrm{~min}$ at a wavelength of $280 \mathrm{~nm}$. Interference sedimentation coefficient distributions, or $\mathrm{C}(\mathrm{M})$, were calculated from the sedimentation velocity data using SEDFIT (Schuck, 2000).

\section{PG preparation}

PG was extracted from the E. coli $\mathrm{DH} 5 \alpha$ strain by following a method described previously (Desmarais et al., 2014). Briefly, cells were cultured until they reached an $\mathrm{OD}_{600}$ of $0.7-0.8$ and then collected at $5,000 \times g, 4^{\circ} \mathrm{C}$. The collected bacteria were dripped into the boiling $6 \%(w / v)$ SDS and stirred at $500 \mathrm{rpm}$ in a boiling water bath for $3 \mathrm{~h}$ before incubating overnight at room temperature. The large $P G$ polymers were collected by ultracentrifugation at $130,000 \times g$ for $1 \mathrm{~h}$ at room temperature and washed repeatedly to remove SDS. The pellet was treated with Pronase $\mathrm{E}(200 \mu \mathrm{g} / \mathrm{mL}$ final concentration) for $3 \mathrm{~h}$ at $60^{\circ} \mathrm{C}$ followed by SDS to remove contaminating proteins and washed three times to remove the SDS by ultracentrifugation. Next, the samples were treated with lysozyme $(200 \mu \mathrm{g} / \mathrm{mL}$ final concentration) for $16 \mathrm{~h}$ at $37^{\circ} \mathrm{C}$. Finally, the purified $P G$ is obtained by treating the samples in a boiling water bath for $10 \mathrm{~min}$ and centrifuging it at $13,000 \times g$ to remove the contaminating lysozyme.

\section{Microscale thermophoresis (MST)}

Purified YfiB wild-type and it mutant $\mathrm{YfiB}^{\mathrm{L43P}}$ were fluorescently labeled using the NanoTemper blue protein-labeling kit according to the manufacturer's protocol. This resulted in coupling of the fluorescent dye NT-495. PG was titrated in 1:1 dilutions starting at $1 \mathrm{mmol} / \mathrm{L}$. To determine of the $K_{d}$ values, $10 \mu \mathrm{L}$ labeled protein was mixed with $10 \mu \mathrm{L} P G$ at various concentrations in Hepes buffer (20 mmol/L Hepes, $200 \mathrm{mmol} / \mathrm{L} \mathrm{NaCl}, 0.005 \%$ Tween-20, pH 7.5). After 10 min of incubation, all binding reaction mixtures were loaded into the MST-grade glass capillaries (NanoTemper Technologies), and thermophoresis was measured with a NanoTemper MonolithNT115 system (20\% light-emitting diode, $20 \%$ IR laser power).

\section{Deletion of the yfiB genes}

The $y f i B$ deletion construct was produced by SOE-PCR (Hmelo et al., 2015) and contained homologous flanking regions to the target gene. This construct was ligated into the $\mathrm{pEX} 18 \mathrm{Gm}$ vector between the HindIII and the Kpnl sites. The resulting vector was then used to delete $y$ fiB by two-step allelic exchange (Hmelo et al., 2015). After being introduced into PAO1 via biparental mating with $E$. coli SM10 ( $\lambda$ pir), single crossovers were selected on Vogel-Bonner Minimal Medium (VBMM), which was used for counter-selection against $E$. coli $(P$. aeruginosa can utilize citrate as a sole carbon source and energy source, whereas $E$. coli cannot), containing $50 \mu \mathrm{g} / \mathrm{mL}$ gentamycin. Restreaking was then performed on no-salt Luria-Bertani (NSLB) agar that contained $15 \%$ sucrose to force the resolution of double crossovers. Deletion of $y f i B$ in the strains was confirmed by colony PCR.

For complementation experiments, yfiB wild-type and L43P mutant genes were cloned into the pJN105 vector via the EcoRI and $X$ bal restriction sites, respectively. The plasmids were then individually transformed into the PAO1 $\triangle y f i B$ strain using the rapid electroporation method described in Choi KH et al. (Choi et al., 2006). Transformants were selected on LB plates containing $50 \mu \mathrm{g} / \mathrm{mL}$ gentamycin. For induction, arabinose was added to a final concentration of $0.2 \%$.

\section{Attachment assays}

The attachment assays were carried out using the MBEC ${ }^{\text {TM }}$ (Minimum Biofilm Eradication Concentration, Innovotech, Inc.) biofilm inoculator, which consists of a plastic lid with 96 pegs and 96 individual wells. The MBEC plates containing $150 \mu \mathrm{L}$ LB medium/well were inoculated with $1 \%$ overnight cultures of the yfiB-L43P strain and incubated overnight at $37^{\circ} \mathrm{C}$ without shaking. VB6, L-Trp and arabinose were added as appropriate. The peg lids were washed with distilled water, and the attached cell material was then stained with $0.1 \%$ crystal violet solution ( $5 \%$ methanol, $5 \%$ isopropanol) before further washing to remove excess dye. The crystal violet was re-dissolved in $20 \%$ acetic acid solution, and the absorbance was measured at $600 \mathrm{~nm}$. Assays were performed with 12 wells/strain and repeated independently for each experiment.

\section{BIAcore analysis}

The interaction kinetics of YfiR with VB6 and L-Trp were examined on a SPR machine Biacore 3000 (GE Healthcare) at $25^{\circ} \mathrm{C}$. The 
Table 1. Data collection, phasing and refinement statistics

\begin{tabular}{|c|c|c|c|c|c|}
\hline Data collection & $\begin{array}{l}\text { YfiB (crystal } \\
\text { form I) }\end{array}$ & $\begin{array}{l}\text { YfiB (crystal } \\
\text { form II) }\end{array}$ & VB6-bound YfiR & Trp-bound YfiR & YfiBL43P-YfiR \\
\hline Space group & $P 21$ & P41 & P43212 & P43212 & $P 1$ \\
\hline Wavelength $(\AA)$ & 1.54187 & 0.9791 & 0.97861 & 0.9791 & 1.10000 \\
\hline Resolution $(\AA \AA)^{a}$ & $\begin{array}{l}50.0-2.15 \\
\quad(2.19-2.15)\end{array}$ & $\begin{array}{l}50.0-2.80 \\
(2.85-2.8)\end{array}$ & $\begin{array}{l}50.0-2.4 \\
\quad(2.44-2.4)\end{array}$ & $\begin{array}{l}50.0-2.5 \\
\quad(2.54-2.5)\end{array}$ & $\begin{array}{l}50-1.78 \\
\quad(1.86-1.78)\end{array}$ \\
\hline \multicolumn{6}{|l|}{ Cell dimensions } \\
\hline$a, b, c(\AA)$ & $\begin{array}{l}65.85,90.45 \\
\quad 66.30\end{array}$ & $\begin{array}{l}46.95,46.95 \\
154.24\end{array}$ & $\begin{array}{l}120.24,120.24 \\
84.99\end{array}$ & $\begin{array}{l}120.88,120.88 \\
88.46\end{array}$ & $\begin{array}{l}49.50,58.57 \\
\quad 69.86\end{array}$ \\
\hline$\alpha, \beta, \gamma\left({ }^{\circ}\right)$ & $90,113.87,90$ & $90,90,90$ & $90,90,90$ & $90,90,90$ & $\begin{array}{l}72.93,96.98 \\
90.19\end{array}$ \\
\hline Unique reflections & $37,625(1866)$ & $8,105(412)$ & $24,776(1202)$ & $23170(1132)$ & $67,774(6615)$ \\
\hline $\mid / \sigma l$ & $19.59(2.62)$ & $12.36(4.15)$ & $20.17(2.4)$ & $39.5(4.68)$ & $17.75(1.89)$ \\
\hline Completeness (\%) & $97.1(95.4)$ & $97.8(100)$ & $99.1(98.8)$ & $99.9(100)$ & $96.5(94.6)$ \\
\hline$R_{\text {merge }}(\%)$ & $6.5(44.5)$ & $14.6(49.7)$ & $8.9(56.8)$ & $9.4(89.2)$ & $5.6(46.3)$ \\
\hline$R_{\text {meas }}(\%)$ & $7.4(51.6)$ & $15.4(52.0)$ & $9.6(61.7)$ & $9.6(90.8)$ & $6.6(55.1)$ \\
\hline $\mathrm{CC} 1 / 2^{\mathrm{b}}$ & 0.747 & 0.952 & 0.899 & 0.974 & 0.849 \\
\hline \multicolumn{6}{|l|}{ Refinement } \\
\hline$R_{\text {work }}(\%)$ & 20.14 & 19.17 & 17.82 & 18.66 & 17.90 \\
\hline$R_{\text {free }}(\%)$ & 26.29 & 26.49 & 19.81 & 23.05 & 20.61 \\
\hline \multicolumn{6}{|l|}{ Average $B$ factors $\left(\AA^{2}\right)$} \\
\hline Protein & 25.54 & 42.70 & 38.68 & 35.03 & 32.54 \\
\hline VB6 & - & - & 44.08 & - & - \\
\hline Trp & - & - & - & 87.51 & - \\
\hline $\mathrm{SO}_{4}{ }^{2-}$ & 37.16 & 66.52 & 51.55 & 41.93 & 45.51 \\
\hline $\mathrm{H}_{2} \mathrm{O}$ & 32.91 & 36.09 & 40.58 & 34.75 & 43.52 \\
\hline \multicolumn{6}{|c|}{ Root mean square deviations } \\
\hline Bond lengths $(\AA)$ & 0.009 & 0.009 & 0.007 & 0.007 & 0.007 \\
\hline Bond angles $\left({ }^{\circ}\right)$ & 1.085 & 1.132 & 1.021 & 0.977 & 1.110 \\
\hline \multicolumn{6}{|l|}{ Ramachandran plot } \\
\hline Most favored (\%) & 92.6 & 87.7 & 96.5 & 98.1 & 94.2 \\
\hline Additionally allowed (\%) & 7.4 & 12.3 & 3.5 & 1.9 & 5.8 \\
\hline Generously allowed (\%) & 0 & 0 & 0 & 0 & 0 \\
\hline Disallowed & 0 & 0 & 0 & 0 & 0 \\
\hline
\end{tabular}

a Numbers in parentheses are for the highest resolution shell.

b The values of $\mathrm{CC} 1 / 2$ are for the highest resolution shell.

running buffer $(20 \mathrm{mmol} / \mathrm{L}$ HEPES, $\mathrm{pH} 7.5,150 \mathrm{mmol} / \mathrm{L} \mathrm{NaCl}$, $0.005 \%(v / v)$ Tween-20) was vacuum filtered, and degassed immediately prior to use. YfiR at $10 \mu \mathrm{g} / \mathrm{mL}$ in $10 \mathrm{mmol} / \mathrm{L}$ sodium acetate ( $\mathrm{pH}$ 5.5) was immobilized to 3000 response units on the carboxymethylated dextran surface-modified chip (CM5 chip). The binding affinities were evaluated over a range of $2.5-40 \mathrm{mmol} / \mathrm{L}$ concentrations. Meanwhile, for both binding assays, the concentration of $10 \mathrm{mmol} / \mathrm{L}$ was repeated as an internal control. All of the data collected were analyzed using BIAevaluation software version 4.1.
ITC assays

ITC experiments were performed in a buffer composed of $20 \mathrm{mmol} / \mathrm{L}$ Tris $(\mathrm{pH} 8.0)$ and $150 \mathrm{mmol} / \mathrm{L} \mathrm{NaCl}$ at $25^{\circ} \mathrm{C}$ using an iTC200 calorimeter (GE Healthcare). YfiB wild-type or its mutants ( $\mathrm{YfiB}^{\mathrm{L} 43 \mathrm{P}}$, $\left.\mathrm{YfiB}^{\mathrm{L} 43 \mathrm{P} / \mathrm{F} 57 \mathrm{~A}}\right)(0.4 \mathrm{mmol} / \mathrm{L}$, in the syringe) was titrated into $\mathrm{YfiR}$ $(0.04 \mathrm{mmol} / \mathrm{L}$, in the cell), respectively. The titration sequence included a single $0.5 \mu \mathrm{L}$ injection, followed by 19 injections of $2 \mu \mathrm{L}$ each, with a 2-min interval between injections and a stirring rate of 
$1000 \mathrm{rpm}$. The calorimetric data were then analyzed with OriginLab software (GE Healthcare).

\section{ACKNOWLEDGMENTS}

We acknowledge the staff at the BL17U and BL18U beamline of the SSRF in China and at the BL-17A beamline of the Photon Factory in Japan for technical assistance during data collection. We thank Y. Y. Chen, Z. W. Yang and B. X. Zhou for help in performing the BIAcore and ITC assays. We also thank Prof. Y. Li (School of Life Sciences, Peking University), L. Jin (School of Life Sciences, Peking University) and Y. Wu (Institute of Microbiology, Chinese Academy of Sciences) for help in performing the MST experiments. This work was supported by grants from the National Natural Science Foundation of China (Grant Nos. 31570768 and 91419308) and the Strategic Priority Research Program (XDB08010301).

\section{ABBREVIATIONS}

c-di-GMP, bis-(3'-5')-cyclic dimeric GMP; CF, cystic fibrosis; DGCs, diguanylate cyclases; EPS, exopolysaccharide; Pal, PG-associated lipoprotein; PDEs, phosphodiesterases; PG, peptidoglycan; VB6, Vitamin B6

\section{COMPLIANCE WITH ETHICS GUIDELINES}

Min Xu, Xuan Yang, Xiu-An Yang, Lei Zhou, Tie-Zheng Liu, Zusen Fan and Tao Jiang declare that they have no conflict of interest. This article does not contain any studies with human or animal subjects performed by the any of the authors.

\section{OPEN ACCESS}

This article is distributed under the terms of the Creative Commons Attribution 4.0 International License (http://creativecommons.org/ licenses/by/4.0/), which permits unrestricted use, distribution, and reproduction in any medium, provided you give appropriate credit to the original author(s) and the source, provide a link to the Creative Commons license, and indicate if changes were made.

\section{REFERENCES}

Adams PD, Afonine PV, Bunkoczi G, Chen VB, Davis IW, Echols N, Headd JJ, Hung LW, Kapral GJ, Grosse-Kunstleve RW et al (2010) PHENIX: a comprehensive Python-based system for macromolecular structure solution. Acta Crystallogr D Biol Crystallogr 66:213-221

Afonine PV, Grosse-Kunstleve RW, Echols N, Headd JJ, Moriarty NW, Mustyakimov M, Terwilliger TC, Urzhumtsev A, Zwart PH, Adams PD (2012) Towards automated crystallographic structure refinement with phenix.refine. Acta Crystallogr D Biol Crystallogr 68:352-367

Beaumont HJ, Gallie J, Kost C, Ferguson GC, Rainey PB (2009) Experimental evolution of bet hedging. Nature 462:90-93
Boehm A, Kaiser M, Li H, Spangler C, Kasper CA, Ackermann M, Kaever V, Sourjik V, Roth V, Jenal U (2010) Second messengermediated adjustment of bacterial swimming velocity. Cell 141:107-116

Caly DL, Bellini D, Walsh MA, Dow JM, Ryan RP (2015) Targeting cyclic di-GMP signalling: a strategy to control biofilm formation? Curr Pharm Des 21:12-24

Camilli A, Bassler BL (2006) Bacterial small-molecule signaling pathways. Science 311:1113-1116

Choi KH, Kumar A, Schweizer HP (2006) A 10-min method for preparation of highly electrocompetent Pseudomonas aeruginosa cells: application for DNA fragment transfer between chromosomes and plasmid transformation. J Microbiol Methods 64:391-397

Desmarais SM, Cava F, de Pedro MA, Huang KC (2014) Isolation and preparation of bacterial cell walls for compositional analysis by ultra performance liquid chromatography. J Vis Exp 83:e51183

Duerig A, Abel S, Folcher M, Nicollier M, Schwede T, Amiot N, Giese $B$, Jenal U (2009) Second messenger-mediated spatiotemporal control of protein degradation regulates bacterial cell cycle progression. Genes Dev 23:93-104

Emsley P, Lohkamp B, Scott WG, Cowtan K (2010) Features and development of Coot. Acta Crystallogr D Biol Crystallogr 66:486-501

Evans TJ (2015) Small colony variants of Pseudomonas aeruginosa in chronic bacterial infection of the lung in cystic fibrosis. Future Microbiol 10:231-239

Giardina G, Paiardini A, Fernicola S, Franceschini S, Rinaldo S, Stelitano V, Cutruzzola $F$ (2013) Investigating the allosteric regulation of YfiN from Pseudomonas aeruginosa: clues from the structure of the catalytic domain. PLoS One 8:e81324

Giddens SR, Jackson RW, Moon CD, Jacobs MA, Zhang XX, Gehrig SM, Rainey PB (2007) Mutational activation of niche-specific genes provides insight into regulatory networks and bacterial function in a complex environment. Proc Natl Acad Sci USA 104:18247-18252

Govan JR, Deretic V (1996) Microbial pathogenesis in cystic fibrosis: mucoid Pseudomonas aeruginosa and Burkholderia cepacia. Microbiol Rev 60:539-574

Grubman A, Phillips A, Thibonnier M, Kaparakis-Liaskos M, Johnson C, Thiberge JM, Radcliff FJ, Ecobichon C, Labigne A, de Reuse $\mathrm{H}$. et al (2010) Vitamin B6 is required for full motility and virulence in Helicobacter pylori. MBio 1

Ha DG, O'Toole GA (2015) c-di-GMP and its effects on biofilm formation and dispersion: a Pseudomonas aeruginosa review. Microbiol Spectr 3, MB-0003-2014

Haussler S, Tummler B, Weissbrodt H, Rohde M, Steinmetz I (1999) Small-colony variants of Pseudomonas aeruginosa in cystic fibrosis. Clin Infect Dis 29:621-625

Haussler S, Ziegler I, Lottel A, von Gotz F, Rohde M, Wehmhohner D, Saravanamuthu S, Tummler B, Steinmetz I (2003) Highly adherent small-colony variants of Pseudomonas aeruginosa in cystic fibrosis lung infection. J Med Microbiol 52:295-301

Hengge R (2009) Principles of c-di-GMP signalling in bacteria. Nat Rev Microbiol 7:263-273

Hickman JW, Tifrea DF, Harwood CS (2005) A chemosensory system that regulates biofilm formation through modulation of 
cyclic diguanylate levels. Proc Natl Acad Sci USA 102:1442214427

Hmelo LR, Borlee BR, Almblad H, Love ME, Randall TE, Tseng BS, Lin C, Irie Y, Storek KM, Yang JJ et al (2015) Precisionengineering the Pseudomonas aeruginosa genome with two-step allelic exchange. Nat Protoc 10:1820-1841

Holm L, Rosenstrom P (2010) Dali server: conservation mapping in 3D. Nucleic Acids Res 38:W545-W549

Huertas MG, Zarate L, Acosta IC, Posada L, Cruz DP, Lozano M, Zambrano MM (2014) Klebsiella pneumoniae yfiRNB operon affects biofilm formation, polysaccharide production and drug susceptibility. Microbiology 160:2595-2606

Hufnagel DA, DePas WH, Chapman MR (2014) The disulfide bonding system suppresses CsgD-independent cellulose production in Escherichia coli. J Bacteriol 196:3690-3699

Jenal U (2004) Cyclic di-guanosine-monophosphate comes of age: a novel secondary messenger involved in modulating cell surface structures in bacteria? Curr Opin Microbiol 7:185-191

Kirillina O, Fetherston JD, Bobrov AG, Abney J, Perry RD (2004) $\mathrm{HmsP}$, a putative phosphodiesterase, and $\mathrm{HmsT}$, a putative diguanylate cyclase, control Hms-dependent biofilm formation in Yersinia pestis. Mol Microbiol 54:75-88

Kirisits MJ, Prost L, Starkey M, Parsek MR (2005) Characterization of colony morphology variants isolated from Pseudomonas aeruginosa biofilms. Appl Environ Microbiol 71:4809-4821

Kulasakara H, Lee V, Brencic A, Liberati N, Urbach J, Miyata S, Lee DG, Neely AN, Hyodo M, Hayakawa Y et al (2006) Analysis of Pseudomonas aeruginosa diguanylate cyclases and phosphodiesterases reveals a role for bis- $\left(3^{\prime}-5^{\prime}\right)$-cyclic-GMP in virulence. Proc Natl Acad Sci USA 103:2839-2844

Laskowski RA, MacArthur MW, Moss DS, Thornton JM (1993) PROCHECK: a program to check the stereochemical quality of protein structures. J Appl Crystallogr 26:283-291

Lebedev AA, Vagin AA, Murshudov GN (2008) Model preparation in MOLREP and examples of model improvement using X-ray data. Acta Crystallogr D Biol Crystallogr 64:33-39

Li S, Li T, Xu Y, Zhang Q, Zhang W, Che S, Liu R, Wang Y, Bartlam M (2015) Structural insights into YfiR sequestering by $Y$ fiB in Pseudomonas aeruginosa PAO1. Sci Rep 5:16915

Malone JG (2015) Role of small colony variants in persistence of Pseudomonas aeruginosa infections in cystic fibrosis lungs. Infect Drug Resist 8:237-247

Malone JG, Jaeger T, Spangler C, Ritz D, Spang A, Arrieumerlou C, Kaever V, Landmann R, Jenal U (2010) YfiBNR mediates cyclic di-GMP dependent small colony variant formation and persistence in Pseudomonas aeruginosa. PLoS Pathog 6:e1000804

Malone JG, Jaeger T, Manfredi P, Dotsch A, Blanka A, Bos R, Cornelis GR, Haussler S, Jenal U (2012) The YfiBNR signal transduction mechanism reveals novel targets for the evolution of persistent Pseudomonas aeruginosa in cystic fibrosis airways. PLoS Pathog 8:e1002760

Matias VR, Al-Amoudi A, Dubochet J, Beveridge TJ (2003) Cryotransmission electron microscopy of frozen-hydrated sections of Escherichia coli and Pseudomonas aeruginosa. J Bacteriol 185:6112-6118

Meroueh SO, Bencze KZ, Hesek D, Lee M, Fisher JF, Stemmler TL, Mobashery S (2006) Three-dimensional structure of the bacterial cell wall peptidoglycan. Proc Natl Acad Sci USA 103:44044409

Navarro MV, Newell PD, Krasteva PV, Chatterjee D, Madden DR, O'Toole GA, Sondermann H (2011) Structural basis for c-di-GMPmediated inside-out signaling controlling periplasmic proteolysis. PLoS Biol 9:e1000588

Otwinowski Z, Minor W (1997) Processing of X-ray diffraction data collected in oscillation mode. Methods Enzymol 276:307-326

Parsek MR, Singh PK (2003) Bacterial biofilms: an emerging link to disease pathogenesis. Annu Rev Microbiol 57:677-701

Parsons LM, Lin F, Orban J (2006) Peptidoglycan recognition by Pal, an outer membrane lipoprotein. Biochemistry 45:2122-2128

Pesavento C, Hengge R (2009) Bacterial nucleotide-based second messengers. Curr Opin Microbiol 12:170-176

Raterman EL, Shapiro DD, Stevens DJ, Schwartz KJ, Welch RA (2013) Genetic analysis of the role of yfiR in the ability of Escherichia coli CFT073 to control cellular cyclic dimeric GMP levels and to persist in the urinary tract. Infect Immun 81:30893098

Reinhardt A, Kohler T, Wood P, Rohner P, Dumas JL, Ricou B, van Delden C (2007) Development and persistence of antimicrobial resistance in Pseudomonas aeruginosa: a longitudinal observation in mechanically ventilated patients. Antimicrob Agents Chemother 51:1341-1350

Ren GX, Yan HQ, Zhu H, Guo XP, Sun YC (2014) HmsC, a periplasmic protein, controls biofilm formation via repression of $\mathrm{HmsD}$, a diguanylate cyclase in Yersinia pestis. Environ Microbiol 16:1202-1216

Romling U, Galperin MY, Gomelsky M (2013) Cyclic di-GMP: the first 25 years of a universal bacterial second messenger. Microbiol Mol Biol Rev 77:1-52

Ross $\mathrm{P}$, Weinhouse $\mathrm{H}$, Aloni $\mathrm{Y}$, Michaeli $\mathrm{D}$, Weinberger-Ohana $\mathrm{P}$, Mayer R, Braun S, de Vroom E, van der Marel GA, van Boom JH et al (1987) Regulation of cellulose synthesis in Acetobacter xylinum by cyclic diguanylic acid. Nature 325:279-281

Ross P, Mayer R, Benziman M (1991) Cellulose biosynthesis and function in bacteria. Microbiol Rev 55:35-58

Sanchez-Torres V, Hu H, Wood TK (2011) GGDEF proteins Yeal, YedQ, and YfiN reduce early biofilm formation and swimming motility in Escherichia coli. Appl Microbiol Biotechnol 90:651-658

Schirmer T, Jenal U (2009) Structural and mechanistic determinants of c-di-GMP signalling. Nat Rev Microbiol 7:724-735

Schuck P (2000) Size-distribution analysis of macromolecules by sedimentation velocity ultracentrifugation and lamm equation modeling. Biophys J 78:1606-1619

Shimazaki J, Furukawa S, Ogihara H, Morinaga Y (2012) L-Tryptophan prevents Escherichia coli biofilm formation and triggers biofilm degradation. Biochem Biophys Res Commun 419:715718

Smith EE, Buckley DG, Wu Z, Saenphimmachak C, Hoffman LR, D’Argenio DA, Miller SI, Ramsey BW, Speert DP, Moskowitz SM et al (2006) Genetic adaptation by Pseudomonas aeruginosa to the airways of cystic fibrosis patients. Proc Natl Acad Sci USA 103:8487-8492

Tamayo R, Tischler AD, Camilli A (2005) The EAL domain protein $\mathrm{VieA}$ is a cyclic diguanylate phosphodiesterase. $\mathrm{J}$ Biol Chem 280:33324-33330 
Ueda A, Wood TK (2009) Connecting quorum sensing, c-di-GMP, pel polysaccharide, and biofilm formation in Pseudomonas aeruginosa through tyrosine phosphatase TpbA (PA3885). PLoS Pathog 5:e1000483

Xu K, Li S, Yang W, Li K, Bai Y, Xu Y, Jin J, Wang Y, Bartlam M (2015) Structural and biochemical analysis of tyrosine phosphatase related to biofilm formation $A(T p b A)$ from the opportunistic pathogen Pseudomonas aeruginosa PAO1. PLoS One 10:e0124330

Yang X, Yang XA, Xu M, Zhou L, Fan Z, Jiang T (2015) Crystal structures of $Y$ fiR from Pseudomonas aeruginosa in two redox states. Biochem Biophys Res Commun 461:14-20 